\title{
O IMPACTO DA CONTAGEM CÍCLICA NA ACURACIDADE DE ESTOQUE: MÚLTIPLOS CASOS EM INDÚSTRIAS DO PARANÁ
}

\section{THE IMPACT OF THE CYCLE COUNTING IN THE INVENTORY ACCURACY: MULTIPLE CASES IN INDUSTRIES OF PARANÁ}

\author{
Everton Drohomeretski* E-mail: everton.drohomeretski@fae.edu \\ Fábio Favaretto** E-mail: fabio.favaretto@unifei.edu.br \\ *FAE Centro Universitário (FAE)/Pontifícia Universidade Católica do Paraná (PUCPR), Curitiba, PR \\ ***Universidade Federal de Itajubá (UNIFEI), Minas Gerais, BH
}

Resumo: $O$ presente artigo objetiva identificar o impacto da contagem cíclica na acuracidade de estoque. Como método de pesquisa foi utilizado múltiplos estudos de caso (sete estudos de caso) em indústrias do Paraná. Como base para a coleta de dados utilizou-se de um protocolo de pesquisa. Já para a análise, foi aplicado o método de análise de conteúdo e a triangulação entre os dados coletados. Como principais resultados, a pesquisa contribui na demonstração da relação entre a contagem cíclica, grau de importância atribuída pela organização, número de itens e o índice de acuracidade obtido. $O$ alto nível de controle dos processos de inventário, aliado a utilização da contagem cíclica, possibilita a eliminação da principal causa da falta de acuracidade de estoque erros nos registros de movimentação de materiais - e, com isso, melhora a eficiência operacional das empresas.

Palavras chave: Acuracidade de estoque. Contagem cíclica. Múltiplos casos.

Abstract: This article aims to identify the impact of cycle counting on inventory accuracy. Multiple case studies were used as research method; these include seven case studies of companies in Paraná. A research protocol was used as the basis for collecting the data. For the data analysis, the content analysis method was used with the triangulation of collected data. For the main results, the study demonstrates the relationship between cycle counting, the level of importance attributed by the organization, the number of items and the rate of accuracy obtained. The high level of control of inventory processes, together with the use of cycle counting made it possible to eliminate the main cause of failure in inventory accuracy - errors in recording the movement of material - and with this, improve the operational efficiency of the companies.

Key-words: Inventory accuracy.Cycle counting. Multiple cases.

\section{INTRODUÇÃO}

Em ambientes considerados competitivos, operar com baixo custo passa a ser uma questão de sobrevivência. Para isso, os vários processos das empresas (produção, logística, desenvolvimento de produtos e outros) necessitam atender os requisitos dos clientes e dos stakeholders. Entre estes requisitos, a logística vem ganhando, a cada dia, maior atenção das empresas (MENEZES et al., 2008). Dentre 
as atividades de destaque atualmente na logística, o gerenciamento e o respectivo controle dos estoques estão cada vez mais evidentes, tanto na área empresarial como na área acadêmica. A atenção tem-se voltado para a logística, principalmente pela crescente pressão por redução de custos, gerada principalmente pelo aumento da concorrência.

Dessa forma, a manutenção de um nível adequado e confiável de estoque pode possibilitar a obtenção de diferencial competitivo e, acima de tudo, a sobrevivência no mercado. Dentro desse contexto, surgem vários fatores que dificultam a redução dos níveis de estoque, por aumentar a insegurança em relação ao atendimento da demanda, dentre eles pode-se destacar:

- a irregularidade da demanda;

- a baixa confiabilidade nas informações provenientes dos fornecedores e do processo produtivo;

- baixa acuracidade de estoque, que traz junto a si níveis extras de estoque, para assegurar a continuidade dos processos sem surpresas.

Assim, informações com níveis aceitáveis de qualidade são requisitos importantes no gerenciamento e controle de estoque. A qualidade da informação (QI) é constituída basicamente da sua função (aplicabilidade), dados e contexto onde será utilizada (LILLRANK, 2003). O grau da qualidade da informação necessária irá depender da sua aplicação, ou seja, do seu uso (FAVARETTO, 2007).

A redução nos níveis de estoque sem um nível satisfatório da QI pode acarretar em uma série de agravantes para os processos internos e externos da organização, além dos respectivos custos relacionados (XU et al., 2012). Dois efeitos que a falta de acuracidade nas informações de estoques geram podem ser caracterizados como o aumento no tamanho do lote, problemas no ressuprimento de materiais e a incerteza do atendimento da demanda (UÇKUN et al., 2008; GEL et al., 2010; NACHTMANN et al., 2010; HARDGRAVE et al. 2011).

Nesta linha, Basinger (2006) e Gel et al. (2010) apresentam que a falta de acuracidade de dados do estoque acarreta na redução do nível de serviço e aumenta os custos relacionados às atividades da logística. Na mesma linha, Huschka (2009) destaca que a precisão dos saldos de estoque é um fator crítico para o bom desempenho das atividades industriais. Já Rekik (2011) aponta que os 
efeitos da falta de acuracidade de estoque gera impacto em vários elos da cadeia de suprimentos, tendo assim um impacto nos fornecedores e nos clientes.

A contagem cíclica é considerada por vários autores como uma importante forma de controle de estoque. Segundo Tersine (1994), esta prática permite acompanhar com frequência as divergências e tomar ações corretivas em um período muito próximo da ocorrência da divergência. Algumas discussões recentes sobre a contagem cíclica podem ser encontradas em: Tersine (1994), Wilson (1995), Raman et al. (2001), Rossetti et al. (2001), Latham (2004), Fernandes e Pires (2005), Rossetti et al. (2007), Huschka (2009) e Gel et al. (2010).

Dentro dessa linha, o presente artigo objetiva analisar o impacto da utilização da contagem cíclica de materiais na acuracidade de estoque. Para isso, dividimos o texto em 6 seções, incluindo esta introdução. Na seção seguinte, apresenta-se o método de pesquisa. Na terceira seção, é apresentada a revisão bibliográfica, enfocando os conceitos das pesquisas de acuracidade de estoque, contagem cíclica e metodologia para a contagem cíclica. Na seção subsequente, é apresentada a descrição da coleta de dados. Em seguida, é realizada a análise dos casos. Por fim, são relatadas as conclusões da pesquisa.

\section{MÉTODO DE PESQUISA}

A base do trabalho constitui-se em duas partes: revisão bibliográfica acerca dos tópicos associados ao tema e pesquisa de campo.

A pesquisa de campo foi realizada por meio de estudos de caso, para isso optou-se pela pesquisa qualitativa que possibilita entre outros a proximidade do pesquisador com os dados a serem coletados. A abordagem qualitativa apresenta um potencial para pesquisas na área de logística (NÄSLUND, 2002). Embora, apenas cerca de $13 \%$ da pesquisas publicadas nos anos de 1998 a 2002, em três dos principais periódicos da área de logística, utilizaram a abordagem qualitativa (SPENS e KOVÁCS, 2006). Já em relação ao propósito da pesquisa, ela é descritiva.

Para Cauchick Miguel (2007), a pesquisa descritiva objetiva o relacionamento entre as variáveis, o que se enquadra com o propósito do estudo: identificar o impacto da contagem cíclica no índice de acuracidade de estoque.

Foram então realizados no ano de 2009 estudos de caso em sete empresas industriais de segmentos variados localizadas no estado do Paraná. Esses estudos Revista Produção Online, Florianópolis, SC, v.13, n. 2, p. 655-676, abr./jun. 2013. 
estão ancorados em entrevistas semiestruturadas que duraram cerca de uma hora e meia cada (totalizando aproximadamente 1.260 minutos) e visitas in loco para a realização da observação direta. Todas as entrevistas foram gravadas com consentimento dos entrevistados. A observação direta foi realizada em forma de processual (do recebimento até a expedição de materiais).

Em relação ao número de casos, a presente pesquisa utilizará de múltiplos casos, mais especificamente sete casos, de forma a tornar possível a comparação entre eles e o referencial teórico (VOSS et al., 2002). Ainda Voss et al. (2002) destaca ainda que em relação ao caso único, o múltiplos casos aumenta a validade externa, pois reduz a possibilidade de apreciações tendenciosas do pesquisador. $\mathrm{O}$ número de casos está fundamentado em Eisenhardt (1989) que sugere uma amostra de 4 a 10 casos. A decisão em parar no sétimo caso foi dada em virtude de que os conjuntos de dados coletados nas sete empresas já apresentavam subsídios para o esgotamento teórico.

Já a unidade de análise para o estudo de caso é o setor industrial que apresente diferentes formas de controle de inventário. O foco industrial é motivado pelo baixo número de pesquisas de acuracidade na área de manufatura, pois boa parte das pesquisas relacionadas a esse tema é direcionada ao segmento varejista e centros de distribuição.

Em relação ao segmento de mercado, a presente pesquisa não visa a identificar características em um segmento de mercado específico, com isso, os critérios para a escolha das empresas a serem pesquisadas são: ser uma empresa de manufatura; e estar adequada em relação à utilização ou não da contagem cíclica. Além, disso o critério de amostragem buscou a identificação de casos divergentes, de forma a permitir a comparação dos impactos na acuracidade de estoque em empresas com diferentes estágios de adoção da contagem cíclica.

Nas empresas, as pessoas entrevistadas foram preferencialmente aquelas ligadas ao controle de estoque, os entrevistados tinham na sua maioria cargos de gerência, coordenação ou supervisão. O número de entrevistas variou conforme a empresa, mas em média foram realizadas duas entrevistas por empresa.

A observação direta, realizada a partir de visitas às empresas visou a identificar características físicas das áreas de recebimento, estocagem, produção e expedição, além de verificar os meios de embalagem e identificação dos materiais. Além disso, foram pesquisados dados secundários como registros de controle de Revista Produção Online, Florianópolis, SC, v.13, n. 2, p. 655-676, abr./jun. 2013. 
estoque, indicadores e procedimentos. Essas fontes forneceram importantes informações complementares.

O roteiro de coleta de dados utilizado na entrevista semiestruturada buscou identificar quatro características:

a) características dos respondentes;

b) caracterização da empresa;

c) identificação das características gerais do controle de estoque;

d) descrição do sistema de contagem cíclica.

A Figura 1 apresenta o mapa mental que direcionou o planejamento, aplicação da pesquisa e análise dos dados da pesquisa.

Figura 1 - Mapa mental da pesquisa

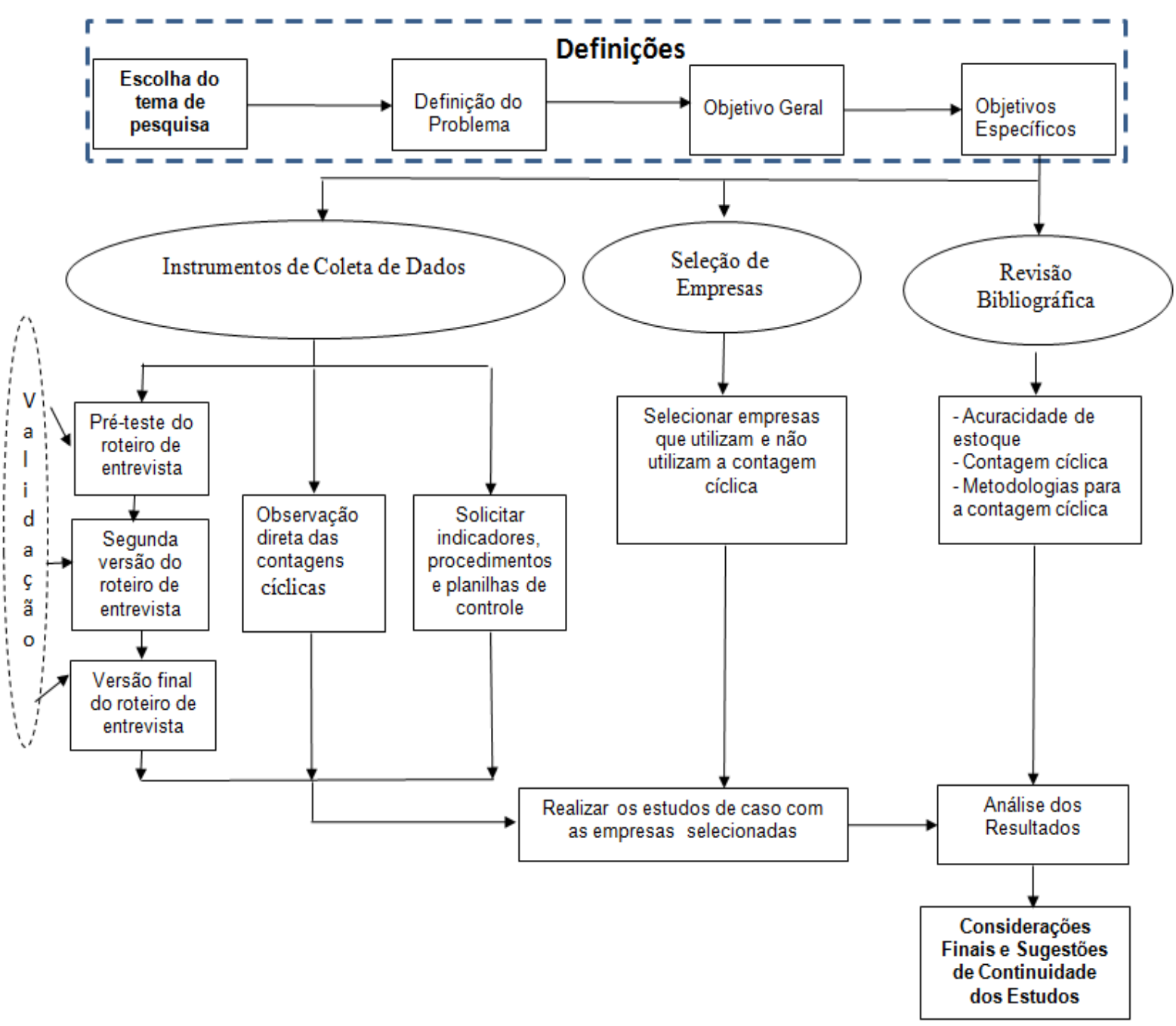

Fonte: Elaborado pelos autores 
Os principais pontos do protocolo de pesquisa utilizado para coletar os dados específicos sobre a contagem cíclica são apresentados abaixo:

a) enfoque: Identificar a sistemática de realização da contagem cíclica;

b) questões a serem levantadas: identificar se a empresa utiliza a contagem cíclica, a metodologia adotada, se tem pessoal dedicado para a atividade, o benefício gerado e se toma ações corretivas;

c) dados coletados: entrevista, análise de documentos e registros de controle da contagem cíclica;

d) análise dos dados: análise da metodologia de contagem cíclica utilizada, comparação do índice de acuracidade entres os casos (empresas que utilizam e não utilizam a contagem cíclica).

A análise dos dados foi realizada por meio da análise de conteúdo, que possibilita a compreensão das características do objeto de estoque e realizar codificações (ANTUNES JUNIOR et al., 2011). A triangulação dos dados coletados foi realizada com base nas entrevistas, observação direta e nos documentos. Além disso, foi realizada a triangulação entre os dados coletados das sete empresas. A triangulação entre os instrumentos de coleta e entre as empresas objetivou aumentar a confiabilidade da pesquisa (BREWER e HUNTER, 2006; MERRIAM, 2009; YIN, 2011).

\section{REFERENCIAL TEÓRICO}

A presente seção objetiva abordar sobre acuracidade de estoque, as principais causas da falta de acuracidade, contagem cíclica e metodologias para contagem cíclica.

\subsection{Acuracidade de estoque}

A acuracidade, do termo inglês accuracy, traz em seu significado a ideia de precisão. A acuracidade de estoque pode ser definida pela mensuração (em percentual) da quantidade de materiais encontrada fisicamente pela quantidade registrada no sistema de informação (SHELDON, 2004). 
O trabalho realizado por Rinehart (1960) pode ser considerado a literatura inicial sobre acuracidade de estoque. Esse trabalho apresenta uma pesquisa em que seu autor analisa os efeitos e as causas das divergências de estoque em uma agência do governo federal americano. Outros autores pioneiros no estudo da acuracidade foram Iglehart e Morley (1972) que, em uma pesquisa realizada no depósito naval americano de Rhode Island, analisaram o impacto da acuracidade no nível de estoque gerado pela insegurança da informação.

A falta de acuracidade de estoque pode impactar de várias maneiras nas operações das empresas, como por exemplo, o aumento no estoque de segurança e por consequência do nível médio de estoque (NACHTMANN et al., 2010). Já Hardgrave et al. (2011) destacam que a manutenção de um índice adequado de acuracidade de estoque é fundamental para a manutenção de um correto sistema de reposição de materiais.

Um exemplo disso, pode ser citado por meio da pesquisa realizada por Pick et al. (2011) em quatro empresas supermercadistas do interior do Rio Grande do Sul. Esses autores identificaram que as redes supermercadistas, que tinham sistemas de informação com melhor nível de implantação e tinham processos rígidos no controle de entrada e saída de mercadoria, apresentaram índices de acuracidade de estoque mais elevados. Por consequência, elas tinham um sistema de reposição mais eficiente.

Em uma pesquisa realizada em unidades industriais, Drohomeretski (2009) apresenta as cinco principais causas da falta de acuracidade de estoque, conforme pode ser verificado na Figura 2. 
Figura 2 - Principais causas da falta de acuracidade de estoque

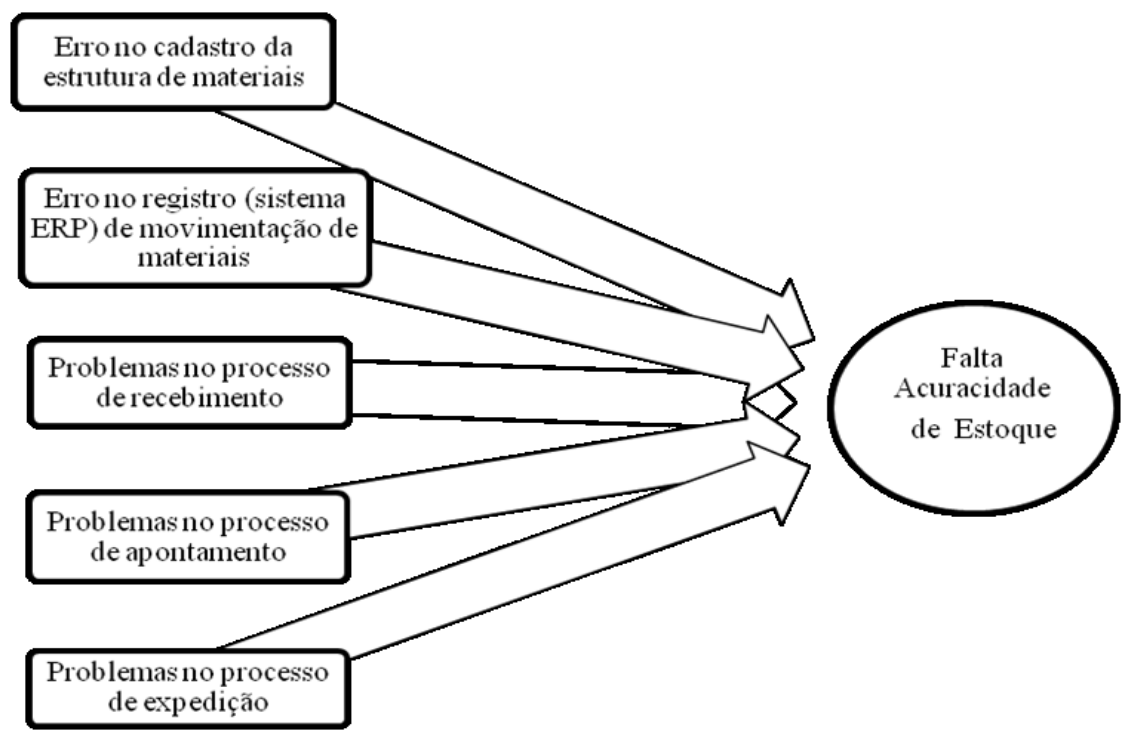

Fonte: Drohomeretski, 2009.

A falta de acuracidade ocorre quando ocorre uma das causas citadas na Figura 2. Por exemplo, quando ocorrem problemas no processo de recebimento, apontamento, expedição ou pelo conjunto de parte ou de todas as causas. Uma das formas de tratar os fatos geradores da falta de acuracidade de estoque é a realização da contagem cíclica de materiais.

\subsection{Contagem Cíclica}

O processo de contagem cíclica de materiais pode ser denominado de várias formas - cycle counting, ciclo de contagem ou inventário cíclico. Para fins de padronização, aos termos utilizados nas publicações internacionais, este trabalho utilizará o termo contagem cíclica (CC).

O controle dos saldos de estoque tem recebido uma atenção cada vez maior das empresas ao longo dos últimos anos, pois a qualidade da informação passou a ser um requisito necessário para tomadas de decisão na logística. Ballard (1996) apresenta a importância de monitorar o estoque em relação ao inventário. Este autor relata que os saldos de estoque podem ser monitorados por meio do inventário periódico - normalmente uma contagem anual dos estoques - ou por meio de verificações contínuas realizando a $\mathrm{CC}$ e a contagem residual do estoque.

A CC é um dos meios utilizados para eliminar a fonte do erro de informação do estoque e aumentar a frequência de auditorias de estoque. As auditorias de 
estoque realizam com frequência determinada a seleção amostral dos itens. Isso minimiza as inconsistências dos registros de inventários nos armazéns das fábricas (NEELEY, 1983; RITZMAN e KRAJEWSKI, 2004; GEL et al., 2010). Nessa mesma linha e de acordo com Bowersox, Closs e Cooper (2007), a acuracidade dos estoques é normalmente mantida pelas contagens periódicas e cíclicas, esta realizada por meio de programação pré-definida e baseada na frequência de movimentação ou no giro de estoque.

De acordo com Gel et al. (2010), a CC é uma sistemática de contagens de materiais realizadas dentro do ano corrente conforme uma programação prédeterminada pela organização. Esta atividade deve acontecer, levando-se em consideração a relevância financeira e a criticidade do material para o processo, sendo que os materiais mais importantes terão uma periodicidade maior de contagem no ano e os itens de menor importância terão uma periodicidade menor. Ainda de acordo com os autores, quanto maior a frequência das contagens maior será a acuracidade de estoque.

Para Tersine (1994), os principais objetivos das CC’s são:

- identificar as causas dos erros;

- corrigir as condições que causam os erros;

- manter um alto nível de acuracidade dos estoques;

- possibilitar a visualização correta dos ativos em estoque.

Com isso, a realização da CC deve ser vista como uma atividade que tem a finalidade de apontar as divergências e encontrar as suas verdadeiras causas (ROSSETTI et al., 2007; GÜMRÜKÇÜ et al., 2008).

Dessa forma, é possível corrigir o processo que está causando a divergência de estoque. Isso pode acontecer através do bom planejamento e operacionalização da CC. Isto é, respeitado o cronograma de contagens e tomando as ações corretivas e preventivas de forma efetiva, teremos uma acuracidade de estoque estabilizada com índices superiores a 99\%. Assim, pode-se eliminar o inventário periódico (contagem geral dos estoques realizado semestralmente ou anualmente) que para a sua realização faz-se necessário interromper as atividades da empresa, horas extras, possíveis perdas de vendas no período, além de outras consequências (RITZMAN e KRAJEWSKI, 2004).

A pesquisa de Iglehart e Morley (1972) objetivava identificar o tipo e a Revista Produção Online, Florianópolis, SC, v.13, n. 2, p. 655-676, abr./jun. 2013. 
frequência de contagem de inventário, de forma a modificar a política de ressuprimento de materiais. Isso para minimizar o custo total do item, em virtude da probabilidade do material estar no armazém com nível abaixo do identificado no sistema de controle. Esses autores buscaram identificar sistemas simples para a implementação de um sistema de controle de estoque. Com isso, Iglehart e Morley (1972) estabeleceram um nível ideal de estoque para proteger das divergências de estoque e ainda determinar uma frequência ótima de contagens que permitissem melhorar a sua acuracidade.

Wilson (1995) apresenta dois objetivos para a realização da CC. O primeiro é alcançar a motivação dos funcionários para diminuir os erros nos registros; o segundo é classificado como operacional, buscando corrigir os erros. O autor ainda relata que o ciclo de contagem é uma espécie de processo de controle da qualidade dos registros de estoque, na ausência da CC os registros de movimentação de materiais tendem a perder qualidade.

Já analisando o impacto da falta de acuracidade na cadeia de suprimentos, Agrawal e Sharda (2012) destacam que a CC auxilia em uma considerável redução de redução de custos na cadeia de suprimentos, tanto no sentido dos fornecedores como no dos clientes.

Nesse sentido, as contagens de estoque devem ser intensificadas e os auditores de estoque não devem se ativer somente a variações financeiras, mas também a variações absolutas - diferença entre quantidade física e do sistema -, ajustando os saldos após as auditorias. Além disso, que sejam realizadas CC que possibilitarão soluções mais rápidas do problema (RAMAN et al., 2001; LATHAM, 2004).

Rossetti et al. (2007) apresentam cinco passos para a realização da CC:
a) escolher os itens a serem contados;
b) preparar o processo de contagem;
c) realizar a contagem;
d) recontar as divergências;
e) identificar e registrar as causas das divergências.

Um caso de sucesso na implantação da CC pode ser verificado no trabalho de Fernandes e Pires (2005). A indústria estudada por estes autores apresentava, antes do início do trabalho de melhoria, uma acuracidade de $62 \%$ para produto 
acabado, causando problemas na programação de produtos, conflitos internos principalmente com as áreas de produção e marketing. Além disso, a baixa acuracidade de estoque causa problemas com os clientes, gerando deméritos para a empresa e a emissão de notas de débito, o que poderia causar empecilhos na produção de novos produtos para os clientes, além, é claro, de prejudicar a imagem da organização perante os seus clientes. Para solucionar o problema, a empresa montou um grupo de trabalho que analisou as principais causas das divergências de estoque e implementou a CC. Após 16 semanas de trabalho, o índice de acuracidade de estoque atingiu $96 \%$, superando o objetivo inicial do projeto que era de $95 \%$ de precisão do estoque de produto acabado.

Ações para melhorar a acuracidade de estoque, como a CC, são cada vez mais abordadas pela literatura acadêmica e pela literatura especializada da área. Um exemplo disso é o estudo realizado por Huschka (2009). Este autor utilizou a simulação em $\mathrm{C}++$ para modelar um sistema de inventário cíclico. O estudo se baseou no controle estatístico do processo (CEP) para tratar as divergências de inventário identificadas ao longo da CC.

Já Gel et al. (2010) em um estudo, por meio de simulação, identificaram que a acuracidade tem impacto direto nos custos de estoque. Os mesmos autores ainda puderam identificar que o aumento na frequência da CC reduz proporcionalmente os custos totais do estoque. Mas, os autores ressaltam que a utilização de tecnologias - como a identificação por radio frequência - em conjunto com a CC geram um impacto mais positivo nos estoques.

\subsection{Metodologias para a contagem cíclica}

De acordo com Rossetti et al. (2001), as metodologias clássicas de determinação da CC são a seleção randômica do ciclo de contagem, ciclo de contagem $\mathrm{ABC}$, processo de controle do ciclo de contagem ciclo baseado na oportunidade, ciclo de contagem baseado nas movimentações de materiais e ciclo de contagem baseado na localização dos materiais. O Quadro 1 apresenta a aplicação de cada uma das metodologias citadas. 
Quadro 1 - Metodologias para a CC

\begin{tabular}{|c|c|c|}
\hline Metodologia & Descrição & Frequência \\
\hline Randômica & Seleção aleatória dos itens de estoque. & $\begin{array}{l}\text { Determinada pelo número total de } \\
\text { itens e o tempo de contagem. }\end{array}$ \\
\hline $\mathrm{ABC}$ & $\begin{array}{l}\text { Baseado na classificação de PARETO, } \\
\text { dedicando maior importância para os itens A, } \\
\text { média importância para os itens B e menor } \\
\text { importância para os itens C. }\end{array}$ & $\begin{array}{l}\text { Quatro contagens para os itens da } \\
\text { classe A, duas para os itens da } \\
\text { classe B e uma contagem dos } \\
\text { itens de classe C. }\end{array}$ \\
\hline Oportunidade & $\begin{array}{l}\text { A seleção é feita com base na facilidade } \\
\text { encontrada no momento da contagem. Por } \\
\text { exemplo, um item que está próximo de } \\
\text { produzir apresenta um menor saldo e por } \\
\text { consequência maior facilidade de contagem. }\end{array}$ & $\begin{array}{l}\text { Estratégia de controle da } \\
\text { organização, dependendo do } \\
\text { processo e do número de itens em } \\
\text { estoque. }\end{array}$ \\
\hline $\begin{array}{l}\text { Movimentação } \\
\text { de estoque }\end{array}$ & $\begin{array}{l}\text { A seleção é feita baseada no número de } \\
\text { movimentações, ou seja, itens com maior } \\
\text { movimentação terão uma frequência maior de } \\
\text { contagem. }\end{array}$ & $\begin{array}{l}\text { Depende do número de itens, } \\
\text { tempo de contagem e volume de } \\
\text { movimentação de cada item. }\end{array}$ \\
\hline Localização & $\begin{array}{l}\text { A seleção é feita por localizações fixas no } \\
\text { armazém, ou seja, a contagem é realizada por } \\
\text { áreas fixas e não por amostras. }\end{array}$ & $\begin{array}{l}\text { Depende do número de itens e } \\
\text { tempo de contagem. }\end{array}$ \\
\hline
\end{tabular}

Fonte: Adaptado de Rossetti et al. (2001)

A decisão pela metodologia a ser adotada vai depender das características dos processos das empresas - número de itens, volume de movimentação, prioridade dos itens no processo, entre outros. Algumas organizações, por exemplo, podem utilizar a combinação de algumas das metodologias (BACKES, 1980).

Para Ballard (1996), a CC pode ser feita baseado no volume de movimentação dos itens ou a contagem pode ser feita de forma aleatória por localização. O autor também sugere calcular o número de contagens por meio da análise do número de erros significantes identificados no inventário periódico, encontrando o número de itens a serem contados periodicamente. O número de itens inventariados é proporcional a taxa de erros encontrados, ou seja, quanto maior a acuracidade esperada, maior deverá ser o número de itens inventariados (WILSON, 1995). No entanto, independente do método escolhido, a contagem deve ser diária, todos os itens do armazém devem ser contados durante o ano e que as divergências encontradas devem ser comunicadas, avaliadas e corrigidas (BALLARD, 1996).

Wilson (1995) apresenta que a precisão dos registros de estoque como um pré-requisito para a implantação e uso de sistemas de controle industrial informatizado, como, por exemplo, a utilização de softwares de MRP. Wilson (1995) Revista Produção Online, Florianópolis, SC, v.13, n. 2, p. 655-676, abr./jun. 2013. 
sugere a aplicação da classificação ABC - classificação de Pareto - para identificar os itens com maior movimento, os quais, segundo o autor, têm maior probabilidade de contagem, identificação e localização.

Ainda em relação a métodos para a implantação da CC, Drohomeretski (2009) propõe um método com cinco etapas sequenciais, conforme a Figura 3. O método envolve desde atividades de planejamento da operação, como a análise das características do processo, até o monitoramento e tomada de ações corretivas.

Figura 3 - Método para implantação da CC

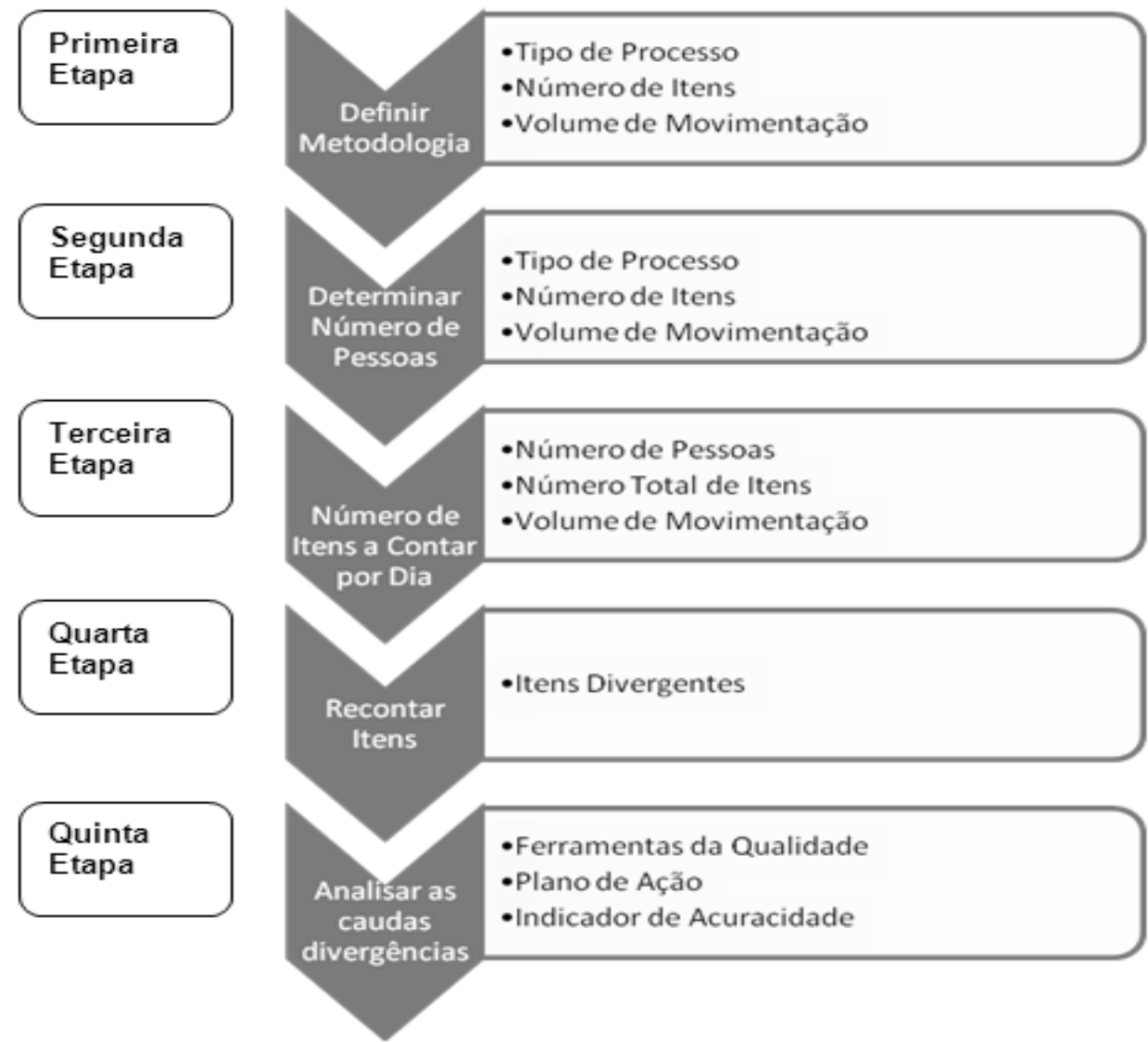

Fonte: Drohomeretski, 2009

Em relação ao índice de tolerância para as divergências de estoque, Sthal (1998) recomenda que sejam analisados os seguintes fatores: valor do material, volume de movimentação do material, lead-time, método de contagem, criticidade do material no processo e impacto na lista de materiais (BOM). De forma mais clara, a APICS (1980) apud Rossetti (2001) quantifica os índices de tolerância em 0,5\% para itens de classe A, 1,0\% para itens de classe B e 5,0\% para itens de classe $C$. 


\section{DESCRIÇÃO DOS CASOS}

Esta seção relata as características gerais do processo de coleta de dados e os estudos de caso em sete indústrias paranaenses. Os estudos de caso foram realizados com base em um roteiro de entrevista, na análise de documentos, nos registros e na observação direta. As entrevistas foram feitas com gerentes ou gestores dos processos que envolvem o inventário. Foram analisados documentos relacionados ao controle de estoque e da acuracidade, principalmente planilhas eletrônicas. Em todas as empresas foram feitas visitas ao chão de fábrica e realizado o acompanhamento de controles e contagens. Foram consultadas 7 empresas industriais para a aplicação dos estudos de caso, denominadas neste trabalho por motivos de sigilo como FP (Fabrica Pesquisada). A Tabela 1 sintetiza as principais características das empresas pesquisadas.

Tabela 1 - Características das empresas pesquisadas

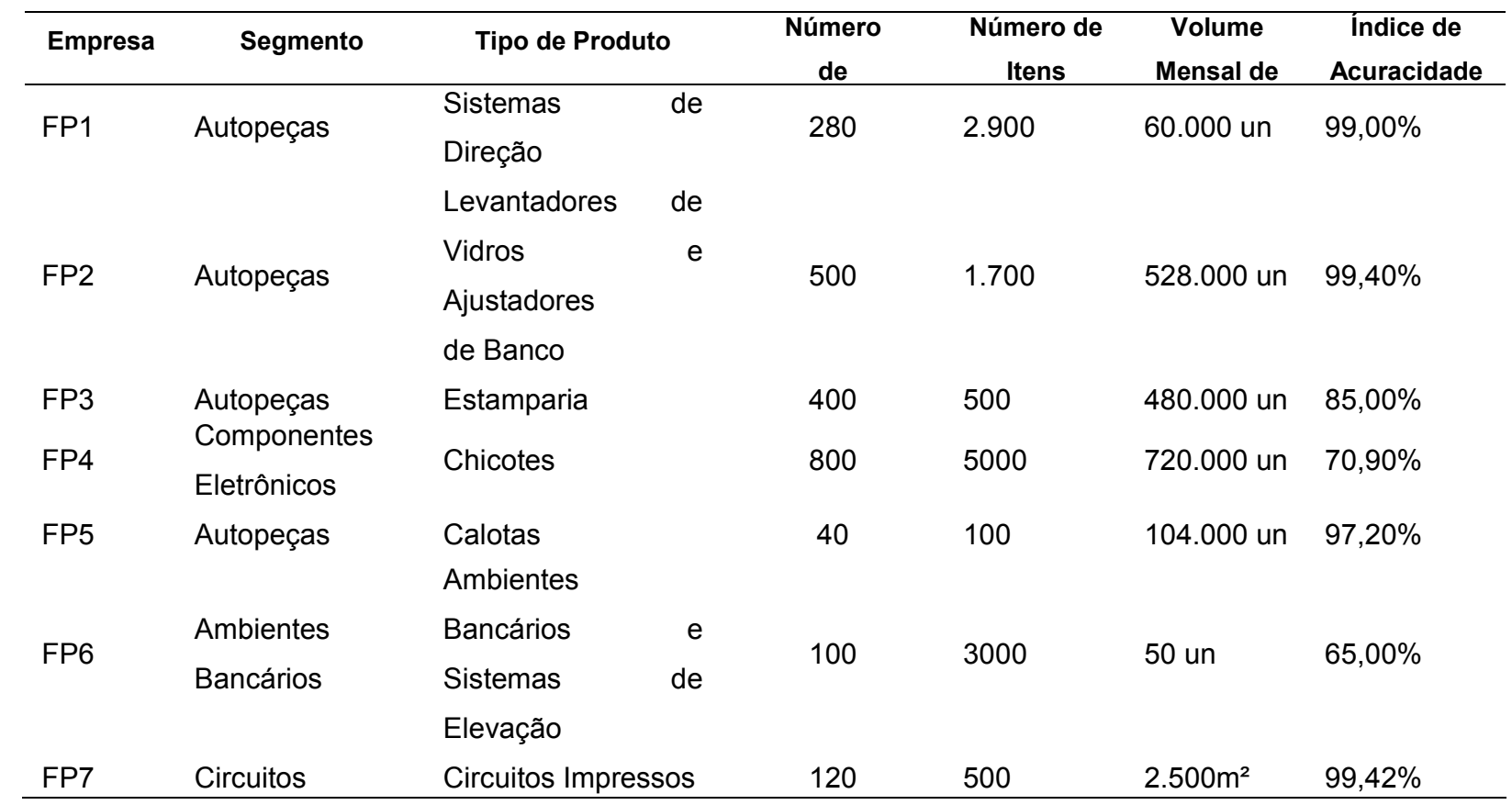

Fonte: Empresas pesquisadas

O critério para a escolha das empresas foi baseado nas seguintes premissas: manter estoque ao longo do processo, controlar o índice de acuracidade de estoque e utilizar ou não a contagem cíclica de materiais. Com isso, a pesquisa não focou determinado segmento de mercado. 
Das sete empresas pesquisadas, seis controlam o índice de acuracidade de estoque. Somente a FP6 não controla regularmente o índice, com isso, os dados para o cálculo do índice foram calculados com base no último inventário realizado. Em relação às três categorias de materiais pesquisadas (matéria-prima, produto em processo e produto acabado), todas as empresas controlam a acuracidade de matéria-prima, somente a FP6 controla produto em processo, e o índice de produto acabado não é controlado somente pela FP4. A Tabela 2 ilustra o índice médio de acuracidade de estoque (IAE médio) dos itens produtivos das fábricas pesquisadas (FP`s).

Tabela 2 - Índice médio de acuracidade de estoque das FP`s

\begin{tabular}{cc}
\hline Empresa & IAE Médio \\
\hline FP1 & $99,99 \%$ \\
FP2 & $99.40 \%$ \\
FP3 & $85,00 \%$ \\
FP4 & $70,90 \%$ \\
FP5 & $97,20 \%$ \\
FP6 & $65,00 \%$ \\
FP7 & $99,42 \%$ \\
\hline Fonte: Registros de controle de acuracidade de estoque das FP`s
\end{tabular}

O roteiro de pesquisa foi desenvolvido com base no referencial teórico, o qual buscou identificar se a empresa utiliza a $\mathrm{CC}$, se tem pessoal dedicado, o nível de importância atribuído, qual a metodologia utilizada para as contagens, se tem hierarquia de contagem e se emite plano de ação para a correção dos desvios. A Tabela 3 ilustra a relação entre os dados coletados com o índice de acuracidade de cada uma das empresas.

Tabela 3 - Análise da utilização da CC

\begin{tabular}{llllllll}
\hline Empresa & $\begin{array}{l}\text { Realiza } \\
\text { CC? }\end{array}$ & $\begin{array}{l}\text { Pessoal } \\
\text { dedicado } \\
\text { à CC? }\end{array}$ & $\begin{array}{l}\text { Importância } \\
\text { Atribuída }\end{array}$ & Metodologia & $\begin{array}{l}\text { Hierarquia } \\
\text { para ajuste } \\
\text { das } \\
\text { divergências }\end{array}$ & $\begin{array}{l}\text { Emite } \\
\text { Plano } \\
\text { de } \\
\text { Ação }\end{array}$ & $\begin{array}{l}\text { IAE } \\
\text { Médio }\end{array}$ \\
\hline FP1 & Sim & Sim & Alta & Randômica & Sim & Sim & $99,99 \%$ \\
FP2 & Sim & Sim & Alta & Randômica & Sim & Sim & $99,40 \%$ \\
FP3 & Sim & Não & Baixa & Oportunidade & Não & Não & $85,00 \%$ \\
FP4 & Sim & Sim & Média & ABC & Sim & Sim & $70,90 \%$ \\
FP5 & Não & Não & - & - & - & Não & $97,20 \%$ \\
FP6 & Não & Não & - & - & - & Não & $65,00 \%$ \\
FP7 & Sim & Não & Alta & ABC & Não & Não & $95,42 \%$ \\
\hline
\end{tabular}

Fonte: Elaborado pelos autores 
Com base nos dados descritos na presente seção, principalmente nas Tabelas 1, 2 e 3 e com o referencial teórico apresentado na terceira seção do presente artigo é possível realizar a análise dos dados pesquisados nas sete empresas.

\section{ANÁLISE DOS DADOS}

O objetivo deste trabalho foi o de analisar o impacto da utilização da contagem cíclica de materiais na acuracidade de estoque. Assim, esta análise procura colaborar em um primeiro momento na utilização da CC e em um segundo momento no impacto desta utilização na acuracidade de estoque.

Inicialmente é analisada a importância da CC na empresa. Com base na Tabela 3, foi possível identificar diferentes níveis de implantação e de importância atribuída para a CC. Na relação entre os dados coletados, é possível identificar que as empresas com melhores índices de acuracidade (FP1 e FP2) utilizam a CC. A seguir é analisada a utilização da CC em cada uma das empresas pesquisadas.

A FP1, apesar de ter iniciado a implantação da CC há pouco tempo, tem alcançado ótimos resultados no controle de inventário (melhorou o índice de acuracidade de matéria-prima de 68\% para 99,7\% e o índice de produto acabado de $91 \%$ para $100 \%$ ). A empresa adota um sistema rígido para os ajustes de inventário, estabelecendo limites de valor para o ajuste, dessa forma quanto maior for a diferença de inventário verificada, maior é o nível hierárquico envolvido. Periodicamente, o setor responsável pelo controle de inventário gera planos de ação e utiliza ferramentas da qualidade para a correção dos desvios, envolvendo vários setores da empresa nas ações corretivas.

Pode-se atribuir parte dos bons resultados verificados na FP1 à importância atribuída pelos gestores da empresa que investem na forma de controle, mantendo pessoal dedicado para a atividade e participam na tomada de ação para a correção das divergências. Conforme se verificou nos registros de inventário, a empresa melhorou o seu índice de 95\% para o índice atual $(99,99 \%)$ apresentado com a implantação da CC.

A FP2 já utilizava a CC como forma de controle há mais tempo que a FP1, no entanto vem aprimorando constantemente essa forma de controle. Um dos pontos de destaque dessa forma de controle na empresa (CC) é o sistema de plano de ação 
adotado, atribuindo metas e controlando a execução das ações corretivas para resolver as divergências de inventário.

Foi possível verificar um grande comprometimento dos envolvidos na operação de controle de inventário. Uma ação interessante e que demonstrou ser efetiva na FP2 foi o estabelecimento de metas de acuracidade, atrelando ao cumprimento da meta uma premiação em dinheiro para os participantes. Com base no indicador de acuracidade de estoque da empresa, é possível evidenciar uma importante melhora no índice. A ação de remunerar os funcionários da logística com um incentivo financeiro é recomendada por Brown et al. (2001) como uma das ações que contribuem para melhorar a acuracidade de estoque.

A FP3, embora tenha um sistema de CC implantado desde 2003, não tem obtido bons índices de acuracidade de estoque. Esta empresa tem passado por um constante crescimento no volume de produção e diminuiu a importância atribuída para o controle de inventário. Até o ano de 2007, a empresa utilizava a metodologia ABC para a realização do CC, contava com duas pessoas para esta atividade e com um sistema de plano de ação implantado. Assim, o índice de acuracidade mantinhase em torno de 99,00\%. Com o crescimento, a empresa passou a utilizar o pessoal dedicado que realizava a CC para executar a operação de expedição. Desta forma, a contagem dos materiais é realizada atualmente conforme os itens apresentam menor quantidade de estoque, ou seja, quando o item está próximo do ponto de produção, os funcionários fazem as contagens (oportunidade de contagem).

A FP4 tem apresentado um caminho contrário ao da FP3. A empresa iniciou de forma efetiva a contatem cíclica, utilizando-se de uma equipe dedicada para a operação há cerca de um ano, a FP4 tem apresentado uma importante melhoria no índice, passando de $54,8 \%$ para $74,9 \%$. Parte desse resultado se deve a implantação de um método rígido de controle das divergências, atrelando planos de ação para a correção dos desvios.

Conforme os registros apresentados pela empresa, a tendência é que o indicador alcance resultados melhores, ou seja, mais próximo a um índice de 95\%. Isso ocorre apesar da complexidade do processo produtivo e do grande número de componentes utilizados, fato que poderia dificultar a atividade de controle de inventário.

Já a FP7 utiliza o CC com o apoio dos funcionários do almoxarifado. As divergências não são tratadas por meio de um plano de ação e sim ajustadas com Revista Produção Online, Florianópolis, SC, v.13, n. 2, p. 655-676, abr./jun. 2013. 
transferências para a produção. Pode-se correlacionar o bom índice de acuracidade com um menor volume de movimentação de materiais em relação às demais empresas pesquisadas.

Em uma análise conjunta das empresas pesquisadas, em relação à importância atribuída para a contagem cíclica, verifica-se que das cinco empresas que utilizam o CC (FP1, FP2, FP3, FP4 e FP7), as empresas que apresentaram melhor índice de acuracidade relataram, ao longo das pesquisas, que a direção da empresa atribui grande importância para a atividade, inclusive no caso da FP1, FP2 e FP4 contando com pessoal exclusivo para a atividade. Ou seja, os gestores entendem que o investimento em pessoal dedicado e qualificado melhora a confiabilidade nos estoques e, por consequência, gera benefícios como redução de custos, aumento na confiabilidade, entre outros. Essa relação pode ser evidenciada na pesquisa de benchmarking realizada por Collins et al. (2001), a qual demonstrou que as empresas, consideradas referência de controle de inventário, dedicam cerca de 3.600 horas/homem por ano e cerca de $5 \%$ do orçamento anual no processo de inventário e mantém um índice de acuracidade de estoque em torno de 99,9\%.

A seguir é feita análise da contribuição da CC na acuracidade de estoques. Em relação à contribuição da utilização de plano de ação com base nas divergências de contagem, das empresas pesquisadas que utilizam a CC, somente três têm um programa implementado de ações corretivas (FP1, FP2 e FP4). Pode-se verificar que as três analisam sistematicamente as causas das divergências de inventário e tomam ações corretivas com frequência. Este fato demonstrou que as três empresas têm melhorado o índice de acuracidade gradativamente. Conclui-se com isso, que a utilização dos dados da CC possibilita identificar as causas das divergências de inventário e tomar as ações corretivas, o que já foi apontado por vários autores, como: Tersine, 1994; Wilson, 1995; Raman et al., 2001; Latham, 2004; Fernandes e Pires, 2005; Rossetti, 2007; Gümrükçü et al., 2008.

\section{CONSIDERAÇÕES FINAIS}

O presente artigo analisou como a utilização da CC interfere no índice de acuracidade de estoque. Foi possível identificar que as maiorias das empresas que utilizam a contagem cíclica apresentaram melhor desempenho no índice de acuracidade de estoque. Por exemplo, a FP1 saltou de um índice de acuracidade

Revista Produção Online, Florianópolis, SC, v.13, n. 2, p. 655-676, abr./jun. 2013. 
que não passava $80 \%$ para um índice de 99,99\%, com uma melhora significativa no controle de matéria-prima. Outro caso que demonstrou resultados significativos com a implantação da CC foi a FP4 que, embora ainda apresente índice bem abaixo dos níveis de classe mundial, melhorou o índice em mais de $30 \%$ em relação ao período em que não realizava a CC. Ainda, pôde-se identificar que o apoio dos gestores é fundamental para que a contagem cíclica seja uma forma de controlar constantemente a acuracidade de estoque. Isso pode ser evidenciado na FP3, que em virtude do aumento no volume de trabalho, passou a ocupar os funcionários dedicados a realizar a CC em outras atividades operacionais. Com isso, a frequência de contagem foi diminuída e as causas não mais foram tratadas formalmente com ferramentas da qualidade. Devido a isso, a empresa apresentou uma importante redução do indicador de acuracidade de estoque: de aproximadamente $99 \%$ para um índice atual de 85\%.

Pôde-se identificar que uma atividade importante para a efetiva aplicação da CC seria a análise das causas das divergências e a aplicação de planos de ação para correção das divergências. Isso demonstra que a CC é um método necessário para identificar falhas nos processos de controle de inventário e corrigi-las. Outro ponto importante evidenciado para o sucesso da CC é o envolvimento dos funcionários da logística no controle de estoque. A importância desse envolvimento foi possível ser evidenciada na FP2, que dá uma gratificação mensal de $\mathrm{R} \$ 90,00$ para os funcionários da logística quando o objetivo do índice de acuracidade é alcançado. Nesse sentido, os entrevistados da FP2 relataram que os funcionários passaram a executar com maior atenção os processos de recebimento, transferências de depósitos, apontamento e expedição.

\section{REFERENCIAS}

AGRAWAL, P. M.; SHARDA, R. Impact of frequency of alignment of physical and information system inventories on out of stocks: A simulation study. International Journal of Production Economics, v. 136, n.1, p. 45-55, 2012.

ANTUNES JUNIOR, J. A. V.; PELLEGRIN, I.; LEIS, R. P.; ZIMMER, M. V.; VACCARO, G. L. R.; LACERDA, D. P. Os processos de aprendizagem organizacional e a inovação: um estudo de caso longitudinal (1986-1995) em uma empresa do setor petrolífero brasileiro. Revista Produção Online, v.11, n. 2, p. 526564, abr./jun., 2011. 
BACKES, R. W. Cycle Counting - A Better Method for Achieving Accurate Inventory Records. Production and Inventory Management. $2^{\circ}$ Quarter, p. 36-44, 1980.

BALLARD, R. L. Methods of inventory monitoring and measurement. Logistics Information Management, v. 9, n 3, p. 11-18, 1996.

BASINGER, K. L. Impact of inaccurate data on supply chain inventory performance. 2006, 207 p. Tese (doutorado) - Programa de Engenharia de Produção de Sistemas, The Ohio State University, 2006.

BOWERSOX, D; CLOSS, D; COOPER, M. Gestão da cadeia de suprimentos e logística. Rio de Janeiro: Elsevier, 2007.

BREWER, J.; HUNTER, A. Foundations of multimethod research: synthesizing styles. Thousabds Oaks: Sage, 2006.

BROWN, K. L; INMAN R. A.; CALLOWAY J. A. Measuring the efects of inventory inaccuracy in MRP inventory and delivery performance. Production Planning \& Control, v. 12, v. 1, p. 46-57, 2001.

COLLINS, T. et al. Benchmarking for Best Practices in Inventory Integrity. In: INDUSTRIAL ENGINEERING RESEARCH CONFERENCE, 2001, Proceedings...Dallas:Texas, 2001.

CAUCHICK MIGUEL, P. A. C. Estudo de caso na engenharia de produção: estruturação e recomendações para sua condução. Revista Produção, v. 17, n. 1, p. 216-229, jan./abr. 2007.

DROHOMERETSKI, E. Um estudo do impacto das formas de controle de inventário na acuracidade de estoque. 2009. 157 p. Dissertação (mestrado) Programa de Pós-Graduação em Engenharia de Produção e Sistemas. Pontifícia Universidade Católica do Paraná, Paraná, 2009.

EISENHARDT, K. M. Building theories from case study research. The Academy of Management Review, v. 14, n. 4, p. 532-550, 1989.

FAVARETTO, F. Melhoria da qualidade da informação no controle da produção: estudo exploratório utilizando Data Warehouse. Revista Produção, v. 17, n. 2, p. 343-353, maio/ago. 2007.

FERNANDES, A. F; PIRES, R. S. I. Impactos da falta de acurácia de estoques e proposições para melhorias: estudo de caso em uma empresa fabricante de autopeças. In: SIMPÓSIO DE ENGENHARIA DE PRODUÇÃO, 12., 2005, Bauru, Anais...Bauru/UNESP, 2005.

GEL, E. S.; ERKIP, N.; THULASEEDAS, A. Analysis of simple inventory control systems with execution errors: economic impact under correction opportunities. International Journal of Production Economics, v. 125, n. 1, p. 153-166, 2010. 
GÜMRÜKÇÜ, S.; ROSSETTI, M. D.; BUYURGAN, N. Quantifying the cost of cycle counting in a two-echelon supply chain with multiple items. International Journal of Production Economics, v. 116, p. 263-274, 2008.

HARDGRAVE, B. C.; GOYAL, S.; ALOYSIUS, J. A. Improving inventory management in the retail store: the effectiveness of RFID tagging across product categories. Operations Management Research, v. 4, n. 1-2, p.6-13, 2011.

HUSCHKA, K. Using statistical process control to monitor inventory accuracy. 2009, 64 p. Dissertação (mestrado) Department of Industrial and Manufacturing Systems Engineering College of Engineering - Kansas State University. Programa de Engenharia de Sistemas Industriais, 2009.

IGLEHART, D.L.; MOREY, R.C. Inventory systems with imperfect asset information. Management Science, v.18, n. 8, p. 388-394, 1972.

LATHAM, B. Cycle Counting: The best way to improve inventory accuracy. Warehousing Fórum, v. 19, n. 12, p. 1-12. nov. 2004.

LILLRANK, P. The quality of information. International Journal of Quality \& Reliability Management, v. 20, n. 6, p. 691-703, 2003.

MENEZES, T. M.; GUIMARAES, M. G.; SELLITTO, M. A. Medição de indicadores logísticos em duas operações de montagem abastecidas por cadeias de suprimentos. Revista Produção Online, v. 8, n.1, 2008.

MERRIAM, S. B. Qualitative Research: a guide to design and implementation. San Francisco: Jossey-Bass, 2009.

NACHTMANN, H.; WALLER, M. A.; RIESKE, D. W. The impact of point-of-sale data inaccuracy and inventory. Journal of Business, v. 31, n. 1, p. 149-158, 2010.

NÄSLUND, D. Logistics needs qualitative research: especially action research. International Journal of Physical Distribution \& Logistics Management, v. 32, n. 5, p. 321-338, 2002.

NEELEY, P. S. A. Framework for Cycle Counting. Production and Inventory Management, v. 24, p.23-32, 1983.

PICK, V. L.; DIESEL, L.; SELLITTO, M. A. Influência dos sistemas de informação na gestão de estoques em pequenos e médios supermercados. Revista Produção Online, v.11, n. 2, p. 319-343, abr./jun. 2011.

RAMAN, A.; DEHORATIUS, N.; TON, Z. Execution the missing link in retail operations. California Management Review, v. 43, n. 3, p. 136-52, 2001.

REKIK, Y. Inventory inaccuracies in the wholesale supply chain. International Journal of Production Economics, v. 133, p. 172-181, 2011. 
RINEHART, R. F. Effects and causes of discrepancies in supply operations. Operations Research, v. 8, n. 4, p. 543-564, 1960.

RITZMAN, L. P.; KRAJEWSKI, L. J. Administração da produção e operações. São Paulo: Prentice Hall, 2004.

ROSSETTI, M. D; COLLINS, T.; KURGUND, R. Inventory cycle counting : a review. The Proceedings of the 2001 Industrial Engineering Reserarch Conference, Dallas, Texas, 2001.

ROSSETTI, M. D.; GUMRUKCU, S.; BUYURGAN, N.; ENGLISH, J. Inventory Accuracy Improvement via in a Two-Echelon Supply Chain. The Proceedings of the 2007 Industrial Engineering Reserarch Conference, Dallas, Texas, 2007.

SHELDON, D. H. Achieving inventory accuracy: a guide to sustainable class a excellence in 120 days. Hardcover: J. Ross Publishing, 2004.

SPENS, K. M; KOVÁCS, G. A content analysis of research approaches in logistics research. International Journal of Physical Distribution \& Logistics Management, v. 36, n. 5, p. 374-390, 2006.

STHAL, R. A. Cycle counting: a quality assurance process. Hospital Material Management, v. 20, n. 2, p. 22-28, ago. 1998.

TERSINE, R. Principles of inventory and materials management, 4. ed. New Jersey: Englewwod Cliffs - Prentice Hall, 1994.

UCKUN, C; KARAESNEN, F; SAVAS, S. Investment in improved inventory accuracy in a decentralized supply chain. International Journal of Production Economics, v.113, p. 546-566, jun. 2008.

VOSS, C.; TSIKRIKTSIS, N.; FROHLICH, M. Case research in operations management. International Journal of Operations \& Production Management, v. 22, n .2, p. 195-219, 2002.

WILSON, J. M. Quality control methods in cycle counting for record accuracy management. International Journal of Operations \& Production Management, v. 15, n. 7, p. 27-39, 1995.

XU, J.; JIANG, W.; FENG, G.; TIAN, J. Comparing improvement strategies for inventory inaccuracy in a two-echelon supply chain. European Journal of Operational Research, v. 221, n.1, p. 213-221, 2012.

YIN, R. K. Qualitative Research from Start to Finish. New York: The Guilford Press, 2011.

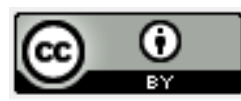

Artigo recebido em 22/02/2012 e aceito para publicação em 27/09/2012. 